\title{
Probabilistic geotechnical engineering analysis based on first order reliability method
}

\author{
Georgios Belokas \\ University of West Attica, Greece \\ gbelokas@uniwa.gr, https:/ /orcid.org/0000-0003-2674-7877
}

\begin{abstract}
Limit equilibrium engineering analysis needs the incorporation of probabilistic approaches for the determination of soil strength statistical measures for deterministic and probabilistic analyses. For the commonly used Mohr-Coulomb strength model, the uncertainty and the characteristic values of cohesion $(c)$ and angle of shearing resistance $(\varphi)$ determination is not straightforward. This paper applies the first order reliability method (FORM) to estimate these values from the direct shear and the typical triaxial tests. The method is verified with test data. Furthermore, the FORM is applied to the planar failure limit equilibrium problem to determine the statistical measures of the safety margin $(S M)$ and safety factor $(S F)$. It is observed that the critical slip surface for the best estimate of the mean $\left(S M_{m}, S F_{m}\right)$, for a $5 \%$ probability of exceedance $\left(S M_{p=5 \%}, S F_{p=5 \%}\right)$ and for the characteristic value $\left(S M_{k}, S F_{k}\right)$ do not coincide. It is interesting that the maximum probability of having a $S M<0$ or $S F<1$ does not correspond to the minimum best estimate of the $S M$ or $S F$. FORM can be a very useful tool for complete probabilistic analyses. Furthermore, probabilistic approaches applied to soil properties estimation can set a framework for the selection of their characteristic values for deterministic analyses.
\end{abstract}

KEYwORDS. Soil strength; Geotechnical engineering; Probabilistic approaches; Reliability; Characteristic values.

\section{OPEN $\bigcirc$ ACCESS}

Citation: Belokas, G., Probabilistic geotechnical engineering analysis based on first order reliability method, Frattura ed Integrità Strutturale, 50 (2019) 354-369.

Received: 16.02 .2019

Accepted: 22.05.2019

Published: 01.10.2019

Copyright: (C) 2019 This is an open access article under the terms of the CC-BY 4.0, which permits unrestricted use, distribution, and reproduction in any medium, provided the original author and source are credited.

\section{INTRODUCTION}

7 he limit equilibrium analysis for the design of geotechnical works requires the application of reliability theory principles for: a) the determination of soil properties in deterministic analyses and b) the uncertainty estimation of the safety margin $(S M)$ or safety factor $(S F)$ in probabilistic analyses. Modern codes of practice (e.g. Eurocode 7 EC7) provide the alternative of both types of analysis, which may include one of the following methods: a) Monte Carlo, b) Point Estimate, and c) First Order Reliability. The application of First Order Reliability Method (FORM) for geotechnical engineering limit states analyses (e.g. $[1,2])$, a method for the estimation of error propagation, has been commonly 
applied to the uncertainty estimation of the experimental results of laboratory tests (e.g. GUM: 1995, see [3]) and can be applied directly wherever a closed form of analytical solution exists.

Under the framework of EC7, the reliability analysis (uncertainty calculation) for a limit equilibrium problem can be performed with respect to the safety margin $(S M)$, which is expected to have a value of $S M \geq 0.0$ for a certain level of confidence. That requires the knowledge of the uncertainty of the parameters that affect the value of $S M$. These parameters normally include the value and the uncertainty of the external and internal loads (permanent and mobile) and of the strength constants, as well as the spatial variability and uncertainty of the model (see Fig.1 based on Kulhawy [4]). One of the most critical components on the overall $S M$ uncertainty is the strength uncertainty both due to spatial variability and model uncertainty. On the other hand, a deterministic analysis requires the best estimate of the loading conditions and the materials properties, which correspond to a specific level of confidence.

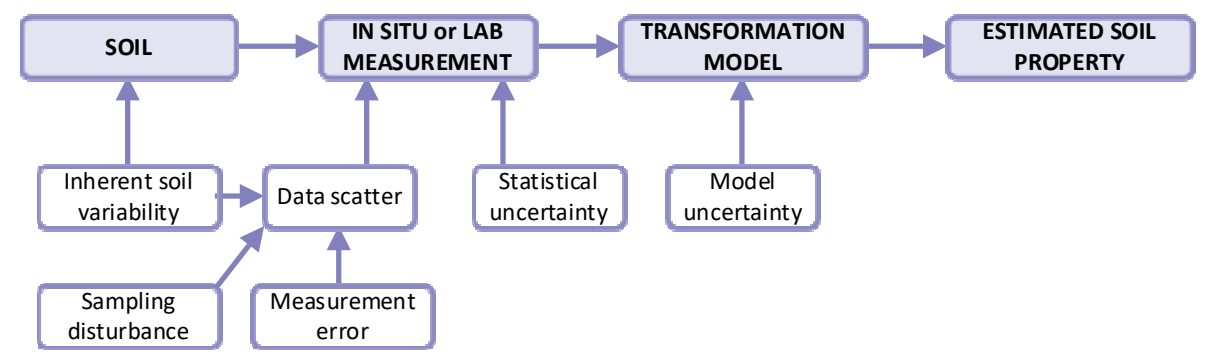

Figure 1: Factors affecting the property uncertainty (based on Kulhawy [4]).

Therefore, for the Mohr - Coulomb strength parameters, cohesion ( $c$ ) and angle of shearing resistance $(\varphi)$, which are used in limit state analysis, an estimation of their mean value and their corresponding variation (or uncertainty) is required. These measures may be calculated either by direct application of statistical methods (e.g. for the direct shear test) or by an error propagation method (e.g. FORM for the typical triaxial test). The present work explores on the application of the FORM for the statistical evaluation of the strength parameters and for the slope stability analytical solution of a wedge failure mechanism. Issues with respect to the design and characteristic strength are also discussed, as well as the capability to apply the FORM into a general limit equilibrium slope stability problem.

\section{STATISTICAL MEASURES OF SOIL PROPERTIES}

A deterministic analysis requires the knowledge of the best estimates of its individual components, i.e. loading conditions and material properties, for a specific confidence level. These correspond to the characteristic values of actions $\left(F_{k}\right)$ and of soil parameters $\left(X_{k}\right)$ defined in EC7 (EN-1997-1). Focusing on soil parameters, for any specific parameter, $X$, that affects the development of the limit state condition, its characteristic value $X_{k}$ is defined as a cautious estimate of the mean value, (i.e. of the best estimate) of the mean, $X_{m}$ (see EN-1997-1). The selection of this characteristic value has to be representative of the volume involved in the considered failure mechanism and it can depend on the type of the failure mechanism (e.g. local vs generalized failure).

When the sample size, $n$, is large enough to apply statistical methods, the characteristic value corresponds to a worse value governing the occurrence of the soil parameter with a calculated probability not greater than $5 \%$ (it is $90 \%$ confidence interval, see EN-1997-1), which for a single variable model is given by Eq.(1).

$$
X_{k}=X_{m}-k \cdot S_{d, X}=X_{k}(1-k \cdot V) \Rightarrow V=S_{d, X} / X_{m}
$$

where $k$ is the confidence level coefficient for a given probability distribution, $S_{d, X}$ is the sample standard deviation and $V$ the variation coefficient. For a specific sample with unknown standard deviation, the $S_{d, X}$ is the corrected standard deviation, which is related to the corrected - unbiased sample variance $\left(s^{2}\right)$ according to Eq.(2).

$$
\operatorname{var}(X)=s_{X}{ }^{2}=\left(S_{d, X}\right)^{2}=\frac{1}{n-1} \sum_{i=1}^{n}\left(X_{i}-X_{m}\right)^{2}
$$


The use of the corrected variance $\left(s_{X}{ }^{2}=\operatorname{var}(X)\right.$, Eq. $\left.(2)\right)$ instead the uncorrected sample variance $\left(\sigma_{X}{ }^{2}\right)$, implies that there is little confidence that $\sigma_{X}{ }^{2}$ is a close estimate of the population variance, $\sigma^{2}$. Had it been $\sigma_{X}^{2} \approx \sigma^{2}$, the sample would closely follow a normal distribution, which is not the case in geotechnical engineering investigations as sample size is usually small, despite that population may follow a normal distribution. Therefore, the direct use of the statistical methods may not be applicable, because they may lead to non-representative values for the soil mass (see [2]).

In order to account for this difficulty on the statistical error, direct values (e.g. [2,5]) or semi-empirical methods (e.g. [5, 6]) have been proposed for the estimation of the variation coefficient $V$. The $V$ coefficient can also be used to include other types of errors, such as the error uncertainty due to ground spatial variability, the measurement error and the transformation uncertainty of the empirical equations application ([6]). However, a good knowledge of the statistical background can provide a better understanding on the selection of the cautious estimate (see [5,7]), or even to apply Bayesian statistical methods (e.g. [2,8]), not only in cases of a small sample but also of complex uncertainties.

Concerning the characteristic values used in deterministic analysis, due to the different types of uncertainties involved (e.g. inherent soil variability, sampling disturbance, Fig.1), engineering judgement is also recommended for their selection (e.g. [9]). Such judgement should evaluate the relative importance of the following uncertainties (see also [6]):

- Sampling quality (sampling type and sample condition).

- The extent of the in-situ and laboratory investigation (the number and spatial distribution of samples and in-situ and laboratory tests).

- The quality of the laboratory tests (accreditation and uncertainty of the laboratory measurements)

- The spatial variability of the parameters and samples distribution with respect to the extent of the considered mechanism of geotechnical soil model.

In addition, the following should also be taken into account: a) existing experience and data on similar soil units (including their uncertainty) and b) the failure mechanism with respect to the geotechnical profile (e.g. generalized vs local failure, short term vs long term conditions, small - large strains).

For a single variable model (e.g. undrained strength, $S_{u}$ ) and a small sample size $(n)$ of laboratory data with unknown standard deviation of the population (the usual case for geotechnical engineering) and assuming a normally distributed population, the resulting estimated distribution follows the Student t-distribution. The estimated characteristic value $\left(X_{k}\right)$, which corresponds to a probability $\mathrm{P}\left(X_{k}<\mu\right)=1-p=1-a / 2$ (i.e. in a certain percentage of the cases the expected value of the true mean, $\mu$, is greater than $X_{k}$ ), is then given by Eq.(3), in which $S E_{X}$ is the sample standard error given by Eq.(4).

$$
\begin{aligned}
& X_{k}=X_{m}-t_{p, n-1} S E_{X}=X_{m}-t_{p, n-1} S_{d, X} / \sqrt{n} \\
& S E_{X}=S_{d, X} / \sqrt{n}
\end{aligned}
$$

where $t_{p, n-1}$ is the $n-1$ degrees of freedom student distribution confidence parameter for the one-sided 1- $p$ lower confidence limit of the true mean $(\mu)$. From Eqs. $(1,3)$ we get the coefficient $k=t_{p, n-1} / n^{0.5}$. Schneider [10] proposed the approximate relationship $X_{k}=X_{m}-0.5 S_{d, X}$, which corresponds to $\mathrm{p}=5 \%$ and $n=14$.

As already mentioned, Eqs. $(3,4)$ correspond to the single variable model (e.g. strength determined from the unconfined compression test, in which $q_{u}=2 S_{u}$, where $S_{u}$ undrained shear strength). The Mohr - Coulomb failure criterion used in limit state analyses, is a two variables linear model that includes two constants (cohesion, $c$, and angle of shearing resistance, $\varphi)$ and two variables. The for the cases of: a) the direct shear test is given by Eq.(5), in which $\sigma_{n}$ is the imposed normal stress (the nonrandom or independent variable) and $\tau$ the measured - observed shear stress (the random or dependent variable) and b) the typical triaxial test is given by Eq.(6), in which $\sigma_{3}$ is the imposed cell pressure - radial stress (the nonrandom or independent variable) and $\sigma_{1}$ the measured - observed vertical stress (the random or dependent variable).

$$
\begin{aligned}
& \tau=c+\sigma_{n} \tan \varphi=a+b \cdot \sigma_{n} \\
& \sigma_{1}=2 c \cdot \cos \varphi /(1-\sin \varphi)+\sigma_{3}(1+\sin \varphi) /(1-\sin \varphi)=a+b \cdot \sigma_{3}
\end{aligned}
$$

With regards to the experiments, if $x_{i}$ is the imposed value and $y_{i}$ is the corresponding observed value of a sample size $n$, the linear model is given by Eq.(7), where $\varepsilon_{i}$ is the error and $a$ and $b$ the model constants. Eq.(7) gives a best estimate of the mean of $y$ for a given $x$. The measurement error $\varepsilon_{i}$ is the deviation of the $y_{i}$ from its best (deterministic) estimate $y=a+x_{i} b$ (Fig.2). In simple linear regression, the best estimate of the constants may be derived from minimizing the error square sum.

$$
y_{i}=a+x_{i} b+\varepsilon_{i}
$$




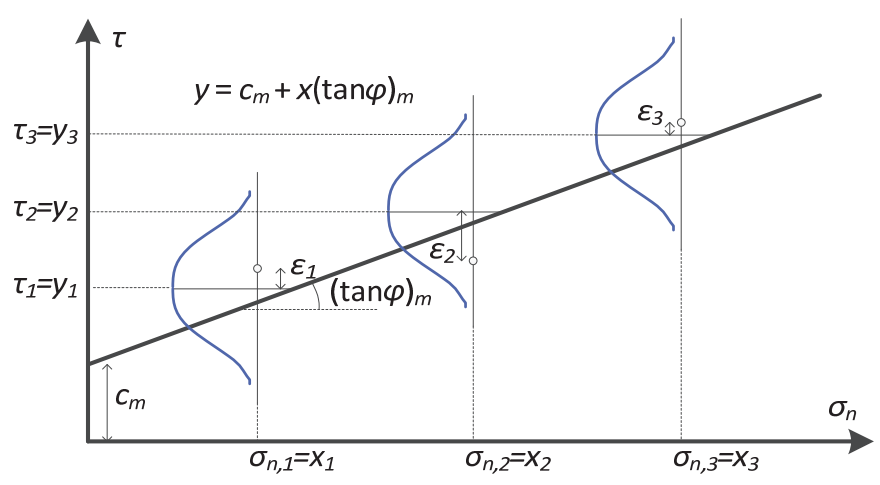

Figure 2: Graphical representation of the regression model.

Assuming that: a) $x_{i}$ is an accurate observation, b) each $x_{i}$ is an independent observation, c) the error $\varepsilon_{i}$ has a constant variation for each $x_{i}$ and $\mathrm{d}$ ) the uncertainties of the $y_{i}$ observations are equivalent (otherwise weight coefficients are required), the best mean estimators for the linear regression coefficients are given by Eqs.(8,9). Observation $x_{i}$ can be a predetermined imposed loading (e.g. the $\sigma_{n}$ in direct shear and the $\sigma_{3}$ in the typical triaxial), while observation $y_{i}$ a measured reaction (e.g. the $\tau$ in direct shear and the $\sigma_{1}$ in the typical triaxial).

$$
\begin{aligned}
& b_{m}=\frac{\sum_{i=1}^{n}\left(x_{i}-\bar{x}\right)\left(y_{i}-\bar{y}\right)}{\sum_{i=1}^{n}\left(x_{i}-\bar{x}\right)^{2}}=\frac{\sum_{i=1}^{n} x_{i} y_{i}-\frac{1}{n} \sum_{i=1}^{n} y_{i} \sum_{i=1}^{n} x_{i}}{\sum_{i=1}^{n} x_{i}^{2}-\frac{1}{n}\left(\sum_{i=1}^{n} x_{i}\right)^{2}}=\frac{\operatorname{Cov}(x, y)}{\operatorname{Var}(x, y)}=r_{x y} \frac{S_{d, y}}{S_{d, x}} \\
& a_{m}=\bar{y}-b_{m} \bar{x}
\end{aligned}
$$

In Eqs. $(8,9)\left(x_{i}, y_{i}\right)$ are the data measurements of the two dimensional sample, $\bar{y}, \bar{x}$ are their mean values, $S_{d, x}$ and $S_{d y}$ are the sample standard deviation of $x$ and $y$ measurements (Eqs. $(10,11))$ and $r_{x y}$ the Pearson sample correlation coefficient (given by Eq.(12)). The $r_{x y}$ is sensitive only to a linear relationship between two variables $\left(\left|r_{x y}\right| \leq 1\right.$, when $r_{x y}=1$ the correlation is a perfect direct, i.e. increasing). Moreover, an unbiased estimate of the variance of $y(x)$ with $\mathrm{n}-2$ degrees of freedom is given by Eq.(13). The standard error estimators $S E_{b}$ and $S E_{a}$ of the $b$ and $a$ regression coefficients are given by Eqs.(14,15), respectively. Some applications in civil and geotechnical engineering of the two variables linear model have been presented by Baecher \& Christian [1], Pohl [8] and Kottegoda \& Rosso [11], as for instance the case of a variation with depth. A classic example is the increasing undrained shear strength with depth. The application of this model in the Mohr - Coulomb strength failure criterion has some individual characteristics that will be presented later.

$$
\begin{aligned}
& S_{d, x}=\sqrt{\frac{1}{n-1} \sum_{i=1}^{n}\left(x_{i}-\bar{x}\right)^{2}} \\
& S_{d, y}=\sqrt{\frac{1}{n-1} \sum_{i=1}^{n}\left(y_{i}-\bar{y}\right)^{2}} \\
& r_{x y}=\frac{n \sum x_{i} y_{i}-\sum x_{i} \sum y_{i}}{\sqrt{\left[n \sum x_{i}^{2}-\left(\sum x_{i}\right)^{2}\right]\left[n \sum y_{i}^{2}-\left(\sum y_{i}\right)^{2}\right]}} \\
& \operatorname{var}(y \mid x)=S=\frac{\sum_{i=1}^{n} \varepsilon_{i}^{2}}{n-2}=S_{d, \varepsilon} \sqrt{\frac{n-1}{n-2}}, \varepsilon_{i}=y_{i}-\hat{a}-\hat{b} x_{i}
\end{aligned}
$$




$$
\begin{aligned}
& S E_{b}=\sqrt{\frac{\operatorname{var}(x \mid y)}{\sum_{i=1}^{n}\left(x_{i}-\bar{x}\right)^{2}}}=\sqrt{\frac{1}{n-2} \frac{\sum_{i=1}^{n} \varepsilon_{i}^{2}}{\sum_{i=1}^{n}\left(x_{i}-\bar{x}\right)^{2}}} \\
& S E_{a}=S E_{b} \sqrt{\frac{1}{n} \sum_{i=1}^{n} x_{i}^{2}}=\sqrt{\frac{1}{n(n-2)}\left(\sum_{i=1}^{n} \varepsilon_{i}^{2}\right) \frac{\sum_{i=1}^{n} x_{i}^{2}}{\sum_{i=1}^{n}\left(x_{i}-\bar{x}\right)^{2}}}
\end{aligned}
$$

\section{STATISTICAL MEASURES FOR THE DIRECT SHEAR TEST - TWO VARIABLES MODEL}

$\mathrm{T}$

he direct shear test gives a direct determination of $c$ and $\tan \varphi$ of the linear Mohr - Coulomb criterion (Eq.(5)), with $\sigma_{n}=x$ and $\tau=y$. For this occasion: a) the best estimates of the mean are $c_{m}=a_{m}$ and $(\tan \varphi)_{m}=b_{m}$ given by Eqs. $(9,8)$, respectively (with $x_{i}=\sigma_{n i}$ and $y_{i}=\tau_{i}$ being the data measurements) and b) the standards error estimators $S E_{(\tan \rho)}=S E_{b}$ and $S E_{c}=S E_{a}$ are given by Eqs.(14,15). Concerning the confidence intervals of the linear regression coefficients estimators, the standard method relies on the normality assumption, which is justified if either: a) the errors in the regression are normally distributed (this leads to a t-statistic) or b) the number of observations $\mathrm{n}$ is sufficiently large (in this case the estimator is approximately normally distributed). Applying a statistical t-test, the linear regression random variables follow a student's t-distribution with $n$-2 degrees of freedom $($ dof $)$, i.e. $T c=\left(c_{m}-c\right) / S E_{c} \sim t_{n-2}, T_{(\tan \varphi)}=\left[(\tan \varphi)_{m}-\tan \varphi\right) / S E_{(\tan \varphi)} \sim t_{n-2}$, where $c$ and $\tan \varphi$ represent the true mean values (or population mean values). The t-test includes the assumptions that the sample is representative of a specific soil unit, the observations are independent, while the variation of $x=\sigma_{n}$ depends only on the uncertainty of the laboratory measurement.

The $\sigma_{n}$ variation has a negligible influence on the total uncertainty. Ignoring the influence of sampling disturbance and spatial variability, the characteristic values of cohesion, $c_{k}$, and angle of shearing resistance, $(\tan \varphi)_{k}$ are given by Eqs. $(16,17)$ and their standard errors by Eqs.(14,15), respectively. The best estimates and their standard errors can be used for probabilistic analyses, by incorporating the standard errors as quantitative measures of the corresponding uncertainties $\left(u_{c}=S E_{\text {tanp }}=S_{d, \text { tanp }} / n^{0.5}\right.$ and $\left.u_{\text {tanp }}=S E_{\text {tanp }}=S_{d, \text { tanp }} / n^{0.5}\right)$.

$$
\begin{aligned}
& c_{k}=c_{m}-t_{p, n-2} S E_{c} \\
& (\tan \varphi)_{k}=(\tan \varphi)_{m}-t_{p, n-2} S E_{(\tan \varphi)}
\end{aligned}
$$

Eqs. $(16,17)$ may be used to give the characteristic Mohr - Coulomb failure envelope: $\tau=c_{k}+\sigma_{n}(\tan \varphi) k$. Alternatively, an estimate of the characteristic failure envelope (see also [1,11]) can be obtained by incorporating the shear stress estimates for a specific probability (given by Eq.(18)). The resulting from Eq.(18) failure envelope is non-linear and needs to be approximated by a linear regression to get the characteristic values of the Mohr - Coulomb failure criterion parameters.

$$
\tau=c_{m}+\sigma_{n}(\tan \varphi)_{m} \pm t_{n-2} \sqrt{\frac{1}{(n-2)}\left(\sum_{j=1}^{n} \varepsilon_{j}^{2}\right)\left[\frac{1}{n}+\frac{\left(\sigma_{n}-\bar{x}\right)^{2}}{\sum_{i=1}^{n}\left(x_{i}-\bar{x}\right)^{2}}\right]}
$$

Table 1 shows a fictional example for the application of the abovementioned relationships for a direct shear test. Typically, three specimens are obtained from each sample, which sample corresponds to a specific depth and location. Applying Eqs. $(8,9)$ we obtain the best estimates of strength parameters and their corresponding uncertainties respectively: $c_{m}=23.95 \mathrm{kPa}$, $(\tan \varphi)_{\mathrm{m}}=0.37950$ (i.e. $\left.\varphi_{m}=20.8^{\circ}\right) S E_{c}=u_{c}=5.3 \mathrm{kPa}$ and $S E_{\text {tan } \varphi}=u_{\text {tan }}=0.01561$. Applying Eqs. $(16,17)$ for a probability $p=5 \%$ we get the following characteristic values: $c_{k 1}=14.5 \mathrm{kPa}$ and $(\tan \varphi)_{k 1}=0.35186$ (see Fig.3, characteristic 1). Applying a linear regression on the characteristic envelope derived from Eq.(18) we get the following characteristic values: $c_{k 2}=16.50 \mathrm{kPa}$ and $(\tan \varphi)_{k 2}=0.38500$ (see Fig.3, characteristic 2), which is less conservative than the characteristic 1. 


\begin{tabular}{cccccccccccccccccc}
\hline Sample & & 1 & \multicolumn{1}{c}{2} & \multicolumn{1}{c}{2} & \multicolumn{1}{c}{3} & \multicolumn{1}{c}{4} & & \multicolumn{1}{c}{5} & \\
Specimen & 1.1 & 1.2 & 1.3 & 2.1 & 2.2 & 2.3 & 3.1 & 3.2 & 3.3 & 4.1 & 4.2 & 4.3 & 5.1 & 5.2 & 5.1 \\
$\sigma_{n}(\mathrm{kPa})$ & 100 & 300 & 500 & 100 & 300 & 500 & 100 & 300 & 500 & 100 & 300 & 500 & 100 & 300 & 500 \\
$\tau(\mathrm{kPa})$ & 56 & 140 & 218 & 56 & 130 & 208 & 69 & 151 & 222 & 48 & 143 & 241 & 76 & 134 & 196 \\
\hline
\end{tabular}

Table 1: Example for direct shear test.

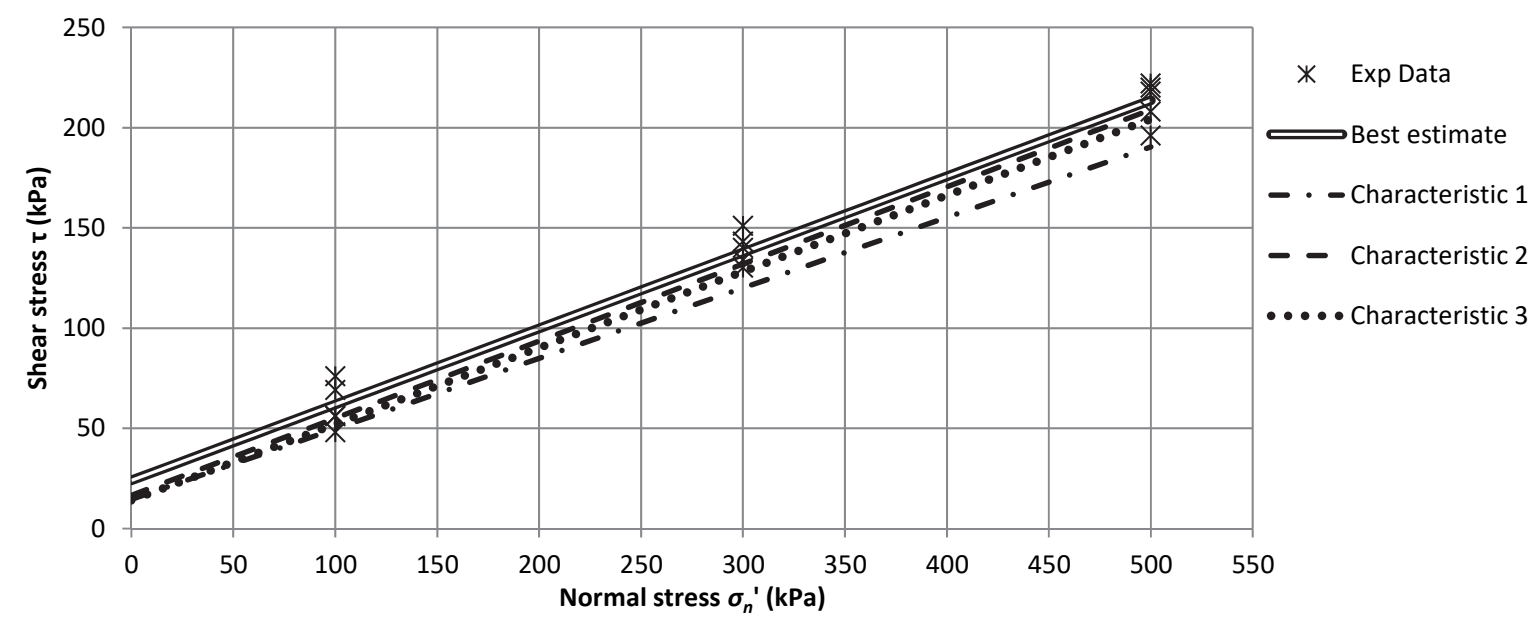

Figure 3: Failure envelopes, best estimate and characteristic envelopes.

The typical test procedure of applying on the three different specimens of each soil sample the $\sigma_{n}=100,300$ and $500 \mathrm{kPa}$ normal stresses respectively, may raise a question concerning the independence of the observations. An alternative is to consider a single variable model for $\tau$ on each one of the three $\sigma_{n}$ values (in our case $n=5$ samples) and then apply a t-test on the observed $\tau$ values, which in our example leads to Table 2. Applying a linear regression on $\sigma_{n}, \tau_{p}=5 \%$ pairs we get the following characteristic values: $c_{k 3}=14.02 \mathrm{kPa}$ and $(\tan \varphi)_{k 3}=0.38056$ (characteristic 3). This approach, overcomes the issues concerning the independence of observation and gives similar results to characteristic 1 (Fig.3), however, it does not give a standard error of the parameters. For this specific case, the maximum standard error on $\tau$ lies close to the previously determined standard error of the cohesion.

\begin{tabular}{cccc}
\hline Specimen & 1 & 2 & 3 \\
$\sigma_{n}(\mathrm{kPa})$ & 100 & 300 & 500 \\
$\tau_{m}(\mathrm{kPa})$ & 61 & 139.6 & 212.8 \\
$S D(\mathrm{kPa})$ & 11.27 & 8.14 & 10.83 \\
$n$ & 5 & 5 & 5 \\
$S E(\mathrm{kPa})$ & 5.0398413 & 3.641428 & 4.841487 \\
$a / 2$ & 0.05 & 0.05 & 0.05 \\
$t_{n-1}$ & -2.131847 & -2.13185 & -2.13185 \\
$\tau_{p=5 \%, \min }$ & 50.255831 & 131.837 & 202.4787 \\
\hline
\end{tabular}

Table 2: Application of $\mathrm{t}$ - test on the values of the observed $\tau$ for each $\sigma_{n}$.

Another approach often used in engineering practice is to apply a t-test on the determined $c$ and $\tan \varphi$ pairs from each sample (again $n=5$ for the examined case). This approach gives $c_{m}=23.95 \mathrm{kPa},(\tan \varphi)_{m}=0.37950$ (i.e. same as before), $S E_{c}=u_{c}=6.6 \mathrm{kPa}$ and $S E_{\text {tanp }}=u_{\text {tan }}=0.02183$ (i.e. greater than before). Applying Eqs. $(15,16)$ for a probability $\mathrm{p}=5 \%$ we get the following cautious estimate of strength parameters: $c_{k 4}=9.8 \mathrm{kPa}$ and $(\tan \varphi)_{k 4}=0.33296$ (characteristic 4), which are unrealistically conservative, as can been in Fig.4, due to the higher uncertainties. 


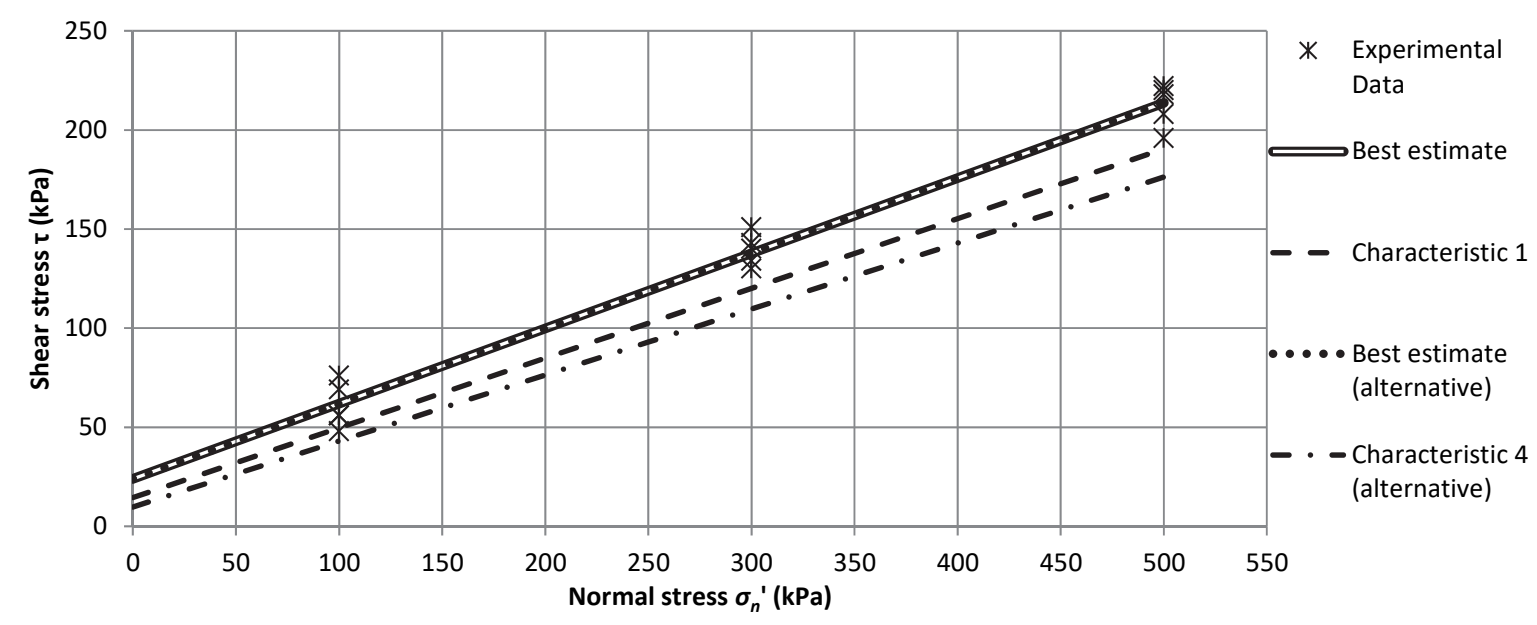

Figure 4: Best estimates and corresponding characteristic envelopes comparison.

\section{STATISTICAL MEASURES FOR THE TYPICAL TRIAXIAL COMPRESSION TEST BY FORM}

I

$\mathrm{n}$ the typical triaxial compression test the Mohr - Coulomb failure criterion parameters are determined indirectly. For this test, a constant horizontal radial stress (the cell pressure) $\sigma_{r}=\sigma_{t}=\sigma_{3}$ is applied to a cylindrical specimen, while the reaction of the axial stress is measured $\left(\Delta \sigma_{a}, \sigma_{l}=\sigma_{3}+\Delta \sigma_{a}\right)$. We can consider that the cell pressure is an accurate observation (i.e. the non - random). The Mohr - Coulomb failure criterion in terms of principal stresses is given by Eq.(6), in which the statistical measures of constants $a$ and $b$ can be determined directly from the two variables models, while the best estimates of the mean for $c$ and $\tan \varphi$ constants are calculated indirectly by the transformation of Eqs.(19,20), respectively.

$$
\begin{aligned}
& \sin \varphi=(b-1) /(1+b) \Rightarrow \varphi=\operatorname{asin}[(b-1) /(1+b)] \text { and } \tan \varphi=\tan \{\operatorname{asin}[(b-1) /(1+b)\}] \\
& c=a(1-\sin \varphi) /(2 \cos \varphi)
\end{aligned}
$$

A way to calculate the uncertainties of $c$ and $\tan \varphi$ is to apply the FORM, which method makes use of the second moment statistics (the mean and the standard deviation) of the random variables and assumes a linearized form of their performance function (e.g. $\left.₹=\mathrm{g}\left(X_{1}, \ldots, X_{n}\right)\right)$ at the mean values of the random variables and independency between all variables. Truncating at the linear terms the Taylor expansion of the performance function about the mean, it is possible to obtain the first order approximation of the variance $\left(\sigma z^{2}\right)$ of the true mean $\left(\mu_{i}\right)$ of $\%$. Assuming uncorrelated non - random variables $X_{1}, \ldots, X_{n}$, the approximation of the variance is given by Eq.(21), an equation commonly used to estimate the uncertainties by error propagation for laboratory tests results.

$$
\sigma_{z}^{2} \approx \sum_{i=1}^{n}\left(\frac{\partial g}{\partial X_{i}}\right)^{2} \operatorname{var}\left(X_{i}\right) \Rightarrow S E_{d, z} \approx \sqrt{\sum_{i=1}^{n}\left(\frac{\partial g}{\partial X_{i}}\right)^{2}\left(S E_{X_{i}}\right)^{2}}
$$

The sample variance $\operatorname{var}\left(X_{i}\right)=s_{X i}^{2}=\left(S_{d, X i}\right)^{2}$ of a $X_{i}$ variable (where $X_{i}$ is $\tan \varphi$ or $c$ ) relates to the standard error $S E_{X i}$ by Eq.(4), while $S E_{X i}$ is a quantitative measure of the corresponding uncertainty $u_{X i}$ (i.e. $u_{X i}=S E_{X i}$ ). The variances $s_{\text {tanp }}{ }^{2}$ and $s_{c}^{2}$ of the Mohr - Coulomb constants may be now calculated by applying Eq.(21) and considering Eqs.(19,20) as the performance functions (i.e. $c=\mathrm{g}(a, \varphi)$ and $\tan \varphi=\mathrm{g}(b)$ or $\varphi=\mathrm{g}(b))$ :

$$
\begin{aligned}
& S E_{\varphi}=\sqrt{\left(\frac{\partial \varphi}{\partial b}\right)^{2}\left(S E_{b}\right)^{2}}, S E_{\tan \varphi}=\sqrt{\left(\frac{\partial \tan \varphi}{\partial b}\right)^{2}\left(S E_{b}\right)^{2}} \\
& S E_{c}=\sqrt{\left(\frac{\partial c}{\partial a}\right)^{2}\left(S E_{a}\right)^{2}+\left(\frac{\partial c}{\partial \varphi}\right)^{2}\left(S E_{\varphi}\right)^{2}}
\end{aligned}
$$


where:

$$
\begin{aligned}
& \frac{\partial \varphi}{\partial b}=\frac{2}{(b+1)^{2} \sqrt{\left.1-(b-1)^{2} /(b+1)^{2}\right)}}, \frac{\partial \tan \varphi}{\partial b}=\frac{2}{(b+1)^{2}\left[1-\frac{(b-1)^{2}}{(b+1)^{3}}\right]^{3 / 2}} \\
& \frac{\partial c}{\partial a}=(1-\sin \varphi) /(2 \cos \varphi) \\
& \frac{\partial c}{\partial \varphi}=a(\sin \varphi-1) /\left(2 \cos ^{2} \varphi\right)
\end{aligned}
$$

Next, the above equations are applied to laboratory results from the Herakleion marl (see Table 3, data from [12]), with 27 specimens and 9 samples ( 3 specimens per sample). Treating each specimen separately (i.e. sample size $n=27$ ), the best estimates of $a$ and $b$ constants of Eq.(6) are determined from the linear regression (Eqs.( 8,9)), which gives $a_{m}=229.6 \mathrm{kPa}$ and $b_{m}=3.02553$. Applying these values into Eqs. $(20,19)$ we get the best estimates $c_{m}=66.0 \mathrm{kPa}$ and $(\tan \varphi)_{m}=0.58225$. The FORM (by application of Eqs. $(23,22,15,14))$ gives the corresponding uncertainties $S E_{c}=u_{c}=15.5 \mathrm{kPa}$ and $S E_{\tan \varphi}=u_{\tan \varphi}=0.0347$. A tstudent distribution for $n-2$ dof (Eqs. $(16,17))$ to account for the error propagation leads to the characteristic values $c_{k 1}=$ $39.60 \mathrm{kPa}$ and $(\tan \varphi)_{k 1}=0.52297$. The best estimate of the mean failure envelope, as well as the estimated characteristic en-

\begin{tabular}{|c|c|c|c|c|c|c|c|c|c|c|c|c|c|c|c|}
\hline Sample & \multicolumn{3}{|c|}{$\Gamma \Sigma 2(5 \mathrm{~m})$} & \multicolumn{3}{|c|}{$\Gamma 1(2 \mathrm{~m})$} & \multicolumn{3}{|c|}{ Г1 (3.5m) } & \multicolumn{3}{|c|}{$\Gamma \Sigma 13(17 \mathrm{~m})$} & \multicolumn{3}{|c|}{ Г1 (18m) } \\
\hline$\sigma_{3}(\mathrm{kPa})$ & 117.72 & 155.98 & 219.74 & 62 & 110 & 304 & 95 & 158 & 383 & 110.85 & 200.12 & 245.25 & 104 & 210 & 427 \\
\hline$\sigma_{1}(\mathrm{kPa})$ & 448.32 & 591.54 & 736.73 & 375 & 606 & 1488 & 475 & 506 & 1383 & 593.51 & 884.86 & 1044.77 & 514 & 785 & 1339 \\
\hline$s(\mathrm{kPa})$ & 283 & 374 & 478 & 219 & 358 & 896 & 285 & 332 & 883 & 352 & 542 & 645 & 309 & 498 & 883 \\
\hline$t(\mathrm{kPa})$ & 165 & 218 & 258 & 157 & 248 & 592 & 190 & 174 & 500 & 241 & 342 & 400 & 205 & 288 & 456 \\
\hline
\end{tabular}
velopes are represented in Fig.5, in which figure the characteristic 2 and 3 envelopes are explained later in the same section. It is evident from Fig. 5 that characteristic 1 can be indeed considered as a conservative estimate of the mean failure envelope.

Table 3.1: Typical triaxial compression data from Herakleion marl ([12]).

\begin{tabular}{cccc|ccc|ccc|cc|c}
\hline Sample & \multicolumn{3}{c|}{$\Gamma 1(6 \mathrm{~m})$} & \multicolumn{3}{|c|}{$\Gamma \Sigma 2(9 \mathrm{~m})$} & \multicolumn{3}{|c|}{$\Gamma \sum 13(24 \mathrm{~m})$} & \multicolumn{2}{c|}{$\Gamma 3(12.5 \mathrm{~m})$} \\
$\sigma_{3}(\mathrm{kPa})$ & 79 & 146 & 265 & 119.68 & 258.98 & 346.29 & 73.57 & 141.26 & 162.85 & 52 & 139 & 222 \\
$\sigma_{1}(\mathrm{kPa})$ & 365 & 618 & 997 & 640.59 & 1056.54 & 1339.07 & 429.68 & 587.62 & 750.47 & 672 & 819 & 1004 \\
$s(\mathrm{kPa})$ & 222 & 382 & 631 & 380 & 658 & 843 & 252 & 364 & 457 & 362 & 479 & 613 \\
$t(\mathrm{kPa})$ & 143 & 236 & 366 & 260 & 399 & 496 & 178 & 223 & 294 & 310 & 340 & 391 \\
\hline
\end{tabular}

Table 3.2: Typical triaxial compression data from Herakleion marl ([12]).

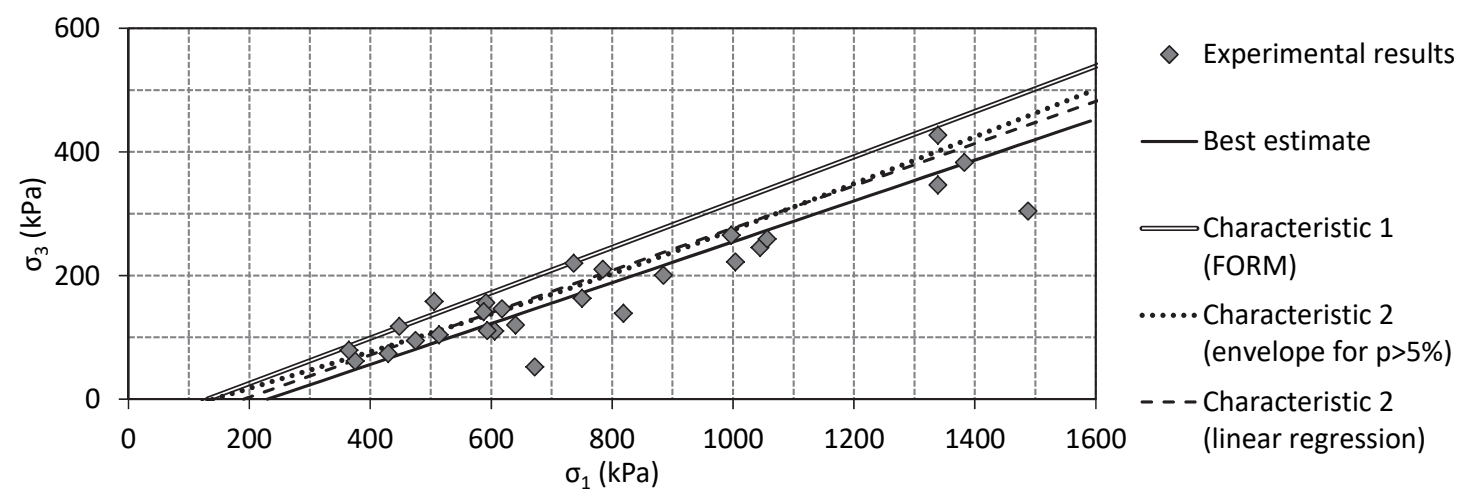

Figure 5: Statistical evaluation of the experimental results of Table 3. 
Treating each sample separately we get the Mohr - Coulomb failure envelope constants of Table 4 (sample size $n=9$ ), which are represented in Fig.6 by the thin coloured lines, in the classic $\tau-\sigma_{n}$ Mohr - Coulomb diagram. Again the remaining characteristic envelopes of Fig. 6 are explained next.

Alternatively, for the characteristic envelope we can apply a t-student distribution into the $y(x)$ estimate (i.e. the predicted $\sigma_{1}$ for given $\sigma_{3}$, given by Eq.(27)) for a $p=5 \%$ probability (i.e. $t_{p, n-2}=1.70814$ ) and $n$ - 2 dof. In Fig. 5 the characteristic 2 line shows the $\sigma_{1}, \sigma_{3}$ graph of the characteristic envelope from Eq.(27) for a $p=5 \%$. This characteristic envelope is non - linear and a linear regression leads to characteristic values $a_{k}=190.36 \mathrm{kPa}$ and $b_{k}=2.92550 \mathrm{for}$ Eq. (5) and then $c_{k 2}=55.6 \mathrm{kPa}$ and $(\tan \varphi)_{k 2}=0.56287$ for the Mohr - Coulomb failure criterion (Figs.(5,6), characteristic 2). Taking into account the results for the direct shear test, it is obvious that Eq.(18) or Eq.(27) systematically gives a more optimistic characteristic envelope than the one computed based on mean values and uncertainty calculation of $c$ and $\tan \varphi$. However, this approach does not take into account the error propagation to calculate the uncertainties.

\begin{tabular}{ccccccccccc}
\hline Sample & Mean & $\Gamma \Sigma 2$ & $\Gamma 1$ & $\Gamma 1$ & $\Gamma \Sigma 13$ & $\Gamma 1$ & $\Gamma 1$ & $\Gamma \Sigma 2$ & $\Gamma \Sigma 13$ & $\Gamma 3$ \\
$c(\mathrm{kPa})$ & 64.34 & 40.99 & 22.34 & 21.24 & 60.41 & 77.75 & 29.69 & 76.83 & 48.20 & 201.58 \\
$\operatorname{Tan} \varphi$ & 0.59415 & 0.53191 & 0.83698 & 0.64201 & 0.64099 & 0.4862 & 0.64606 & 0.59134 & 0.63149 & 0.34038 \\
$\varphi\left({ }^{\circ}\right)$ & 30.42 & 28.01 & 39.93 & 32.70 & 32.66 & 25.93 & 32.86 & 30.60 & 32.27 & 18.78 \\
\hline
\end{tabular}

Table 4: Application of $\mathrm{t}$ - test on the values of the observed $\tau$ for each $\sigma_{n}$.

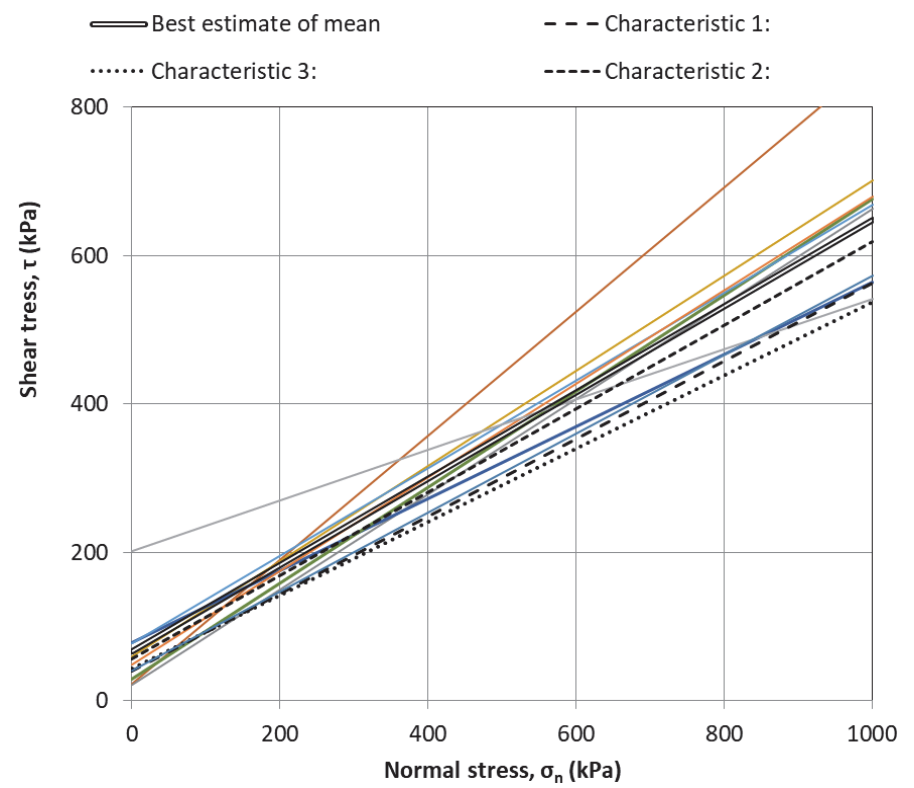

Figure 6: Characteristic and best estimate Mohr - Coulomb failure envelopes superimposed on the Mohr - Coulomb failure envelopes of each sample.

$$
\sigma_{1}=a_{m}+\sigma_{3} b_{m} \pm t_{n-2} \sqrt{\frac{1}{(n-2)}\left(\sum_{j=1}^{n} \varepsilon_{j}^{2}\right)\left[\frac{1}{n}+\frac{\left(\sigma_{3}-\bar{\sigma}_{3}\right)^{2}}{\sum_{i=1}^{n}\left(\sigma_{3 i}-\bar{\sigma}_{3}\right)^{2}}\right]}
$$

An alternative for the characteristic envelope is to compute first the characteristic values of $a$ and $b$ constants by applying a t-student distribution (i.e. $a_{k}=a_{m}-t_{p, n-2} S E_{a}$ and $b_{k}=b_{m}-t_{p, n-2} S E_{b}$ ) for $p=5 \%$ and $n-2$ dof and then apply Eqs. $(20,19)$ to compute the Mohr - Coulomb characteristic constants $c_{k}$ and $(\tan \varphi)_{k}$. This approach, which does not take into account the error propagation, leads to $a_{k}=139.86 \mathrm{kPa}$ and $b_{k}=2.58981$ constants of Eq.(6) (Fig.5, characteristic 3) and to $c_{k 3}=43.4 \mathrm{kPa}$ and $(\tan \varphi)_{k 3}=0.493949$ constants of the Mohr - Coulomb failure criterion (Fig.6, characteristic 3). 
Finally, as it was applied for the direct shear test, a common approach in engineering practice for the estimation of all statistical measures of $c$ and $\tan \varphi$ is first to derive the Mohr - Coulomb constants $(c$, $\tan \varphi)$ for each sample separately (see Table 4) and then apply a statistical t-test on each constant independently for $n / 3-1$ dof (where $n$ the complete number of specimens and $n / 3$ the number of specific locations of soil sampling). For the Herakleion marl case the $n / 3-1=8$ dof lead to: a) mean values: $c_{m}=64.34 \mathrm{kPa},(\tan \varphi)_{m}=0.59415$, b) standard deviation: $S_{d, c}=55.69 \mathrm{kPa}, S_{d, \tan \varphi}=0.13577$, c) standard error:

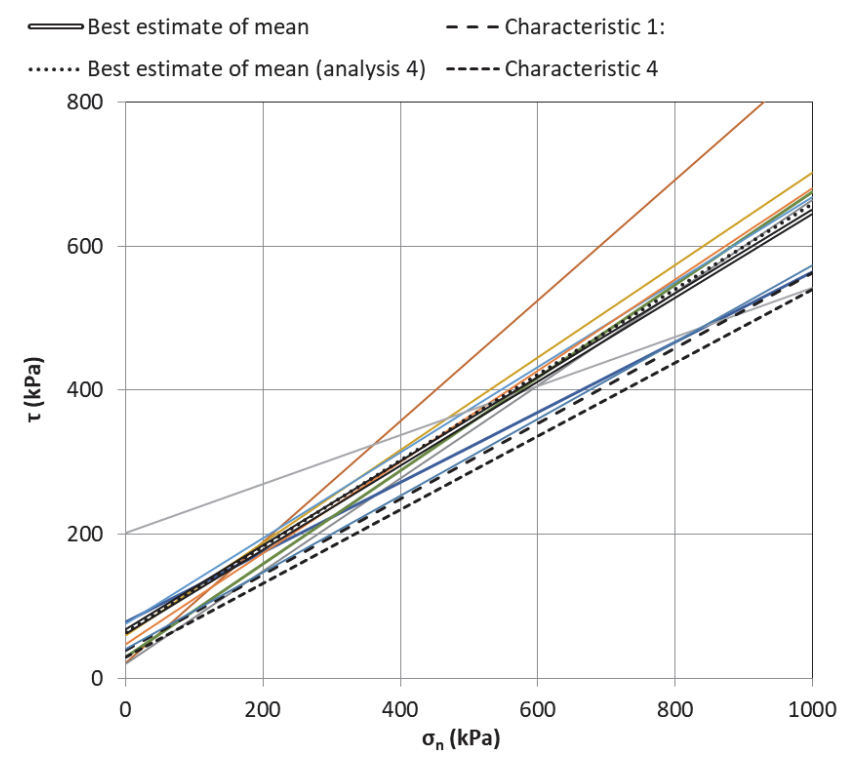

Figure 7: Characteristic and best estimate Mohr - Coulomb failure envelopes superimposed on the Mohr - Coulomb failure envelopes of each sample.

\section{Best estimate}

$a_{m}, b_{m}$ from linear regression of all $\sigma_{1}, \sigma_{3}$ data points

$c_{m},(\tan \varphi)_{m}$ from Eqs. $(20,19)$

$S E_{c}$ and $S E_{\text {tanc }}$ from FORM error propagation

(this gives characteristic 1)

$c_{m},(\tan \varphi)_{m}$ from mean values of Table 4

$S E_{c}$ and $S E_{\text {tan } \varphi}$ from single variable model

(this gives characteristic 4)

$c_{m}(\mathrm{kPa}) \quad(\tan \varphi)_{m} \quad S E_{c}(\mathrm{kPa}) \quad S E_{t a n}(\varphi)$

$\begin{array}{lll}66.00 & 0.58225 & 15.50\end{array}$

0.0347

$\begin{array}{lll}64.34 & 0.59415 & 18.56\end{array}$

0.0423

Table 5: Mohr - Coulomb failure criterion constants (mean values and uncertainties).

\begin{tabular}{|c|c|c|}
\hline Characteristic values & $c_{k}(\mathrm{kPa})$ & $(\tan \varphi)_{k}$ \\
\hline $\begin{array}{l}\text { Characteristic } 1 \text { (SE from FORM error propagation): } \\
c_{k}=c_{m}-t_{p, n-2} S E_{c}, \tan (\varphi)_{k}=\tan (\varphi)_{m-m}-t_{p, n-2} S E_{t a n(\varphi)}\end{array}$ & 39.60 & 0.52297 \\
\hline $\begin{array}{l}\text { Characteristic } 2 \text { : } \\
a_{k}, b_{k} \text { from linear regression on } \sigma_{1 p r e d}, \sigma_{3} \text { data points }\left(\sigma_{1 p r e d}=a_{m}+\sigma_{3} b_{m} \pm t_{n-2} S E_{\sigma 1}\right) \\
c_{k},(\tan \varphi)_{k} \text { from Eqs. }(20,19)\end{array}$ & 55.65 & 0.56288 \\
\hline $\begin{array}{l}\text { Characteristic 3: } \\
a_{k}=a_{m}-t_{, n-2} 2 E_{a}, b_{k}=b_{m}-t_{t, n-2} S E_{b} \\
c_{k,}(\tan \varphi)_{k} \text { from Eqs. }(20,19)\end{array}$ & 43.43 & 0.49395 \\
\hline $\begin{array}{l}\text { Characteristic } 4 \text { (SE from one variable model, Eq. (4), for } c \text { and } \tan \varphi \text { ): } \\
\mathrm{cm} \text { and } \tan \varphi \text { from mean values of Table } 4 \\
c_{k}=c_{m}-t_{t, n-2} S E_{c}, \tan (\varphi)_{k}=\tan (\varphi)_{m}-t_{t, n-2} S E_{\tan (\varphi)}\end{array}$ & 29.82 & 0.50990 \\
\hline
\end{tabular}

Table 6: Mohr - Coulomb failure criterion constants (characteristic values). 
$S E_{c}=18.56 \mathrm{kPa}, S E_{\text {tan } \varphi}=0.04526$ and d) characteristic values: $c k_{4}=29.82 \mathrm{kPa},(\tan \varphi)_{k 4}=0.5099$. The mean value from this alternate approach (characteristic 4, Fig.7) is almost the same with the previously calculated. Fig.7 compares the character istic value from this approach (characteristic 4) with the characteristic 1 from the FORM. The alternate approach is very conservative because it gives a higher SE than the FORM. All the above results are summarized into Tables 5 for the mean values and the corresponding uncertainties and Table 6 for the characteristic values.

\section{APPLICATION OF THE FORM TO A SIMPLE PLANAR FAILURE PROBLEM}

$\mathrm{F}$

urther extending the FORM application to engineering problems calculations, a limit state analysis may be performed in terms of either the safety margin $S M=R-E$ ( $R$ is resistance and $E$ action as defined in EC7) in terms of the safety factor $S F=R / E$. More specifically, in FORM applications the $S M$ application is preferable compared to the $F S$ because the actions $E$ in the denominator of the $S F$ enhances the non-linearity effects in the error propagation. For the safety margin the reliability index is $\beta=S M_{m} / S_{d, S M}$, where $S M_{m}$ is the best estimate of the mean and $S_{d, S M}$ the standard deviation, while by definition it is $\beta_{S M}=1 / V_{S M}$, where $V_{S M}$ the variation coefficient. Since resistances and actions describe different types of random variables, they are expected to be uncorrelated, and their covariance, $\varrho_{R, D}$, can be considered zero. Moreover, the variation of permanent loads is generally small compared and should not greatly affect the $S M$ or $S F$ value. The FORM is applied herein to a planar failure problem, which can be adapted to any type of failure surface (e.g. [13]) of limit equilibrium problems (e.g. method of slices). For the planar wedge failure problem considered herein Fig. 8 shows the geometry. $W$ is the weight of the wedge, $H$ is the height of the slope, $\beta$ is the angle of the slope to the horizontal, $\theta$ is the angle of the plane of failure with respect to horizontal and $N$ and $T$ are the normal and shear reaction forces on the plane of failure. The safety margin is then determined from the equilibrium equations that lead to Eq.(28). On the other hand the $S F$ is given by Eq.(29).

$$
\begin{aligned}
& S M=\left[\mathrm{c} \cdot H+\frac{1}{2} \gamma H^{2} \sin (\beta-\theta)(\cos \theta \tan \varphi-\sin \theta) / \sin \beta\right] / \sin \theta \\
& S F=[2 \mathrm{c} / \gamma+H \sin (\beta-\theta)(\cos \theta \tan \varphi) / \sin \beta] /[H \sin (\beta-\theta) \sin \theta / \sin \beta]
\end{aligned}
$$

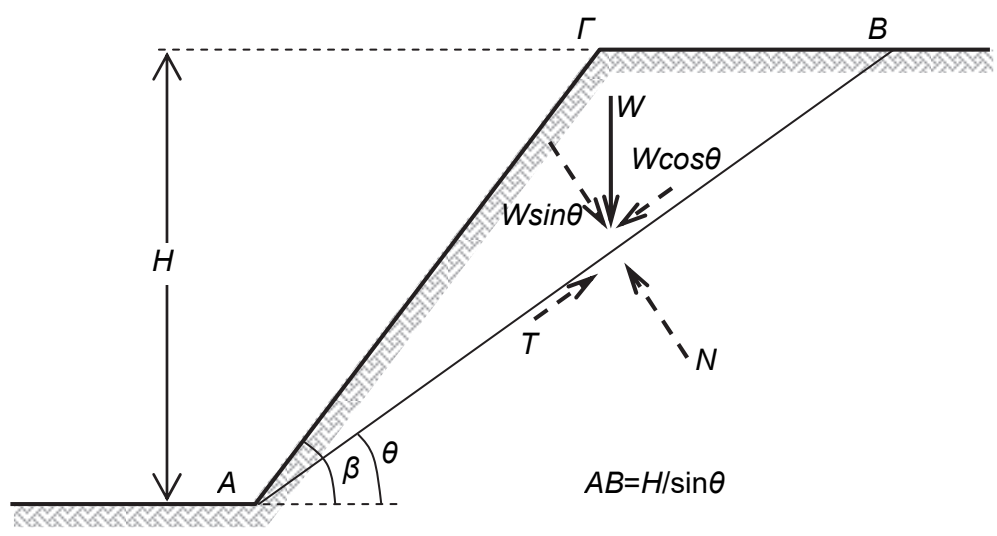

Figure 8: Geometry of two dimensional planar failure.

Applying the FORM on Eq.(28) the standard deviation of the $S M$ is given by Eqs 30 to 33, in which $u_{c}$, $u_{\text {tanp }}$ and $u_{\gamma}$ are the uncertainties of $c, \tan (\varphi)$ and $\gamma$ respectively. Likewise, applying the FORM on Eq.(29) the standard deviation of the SF is given by Eqs.(34-37).

$$
\begin{aligned}
& u(S M)=\sqrt{\left(\frac{\partial S M}{\partial c}\right)^{2} u_{c}^{2}+\left(\frac{\partial S M}{\partial \tan \varphi}\right)^{2} u_{\tan \varphi}^{2}+\left(\frac{\partial S M}{\partial \gamma}\right)^{2} u_{\gamma}^{2}} \\
& \frac{\partial S M}{\partial c}=\frac{H}{\sin \theta}
\end{aligned}
$$




$$
\begin{aligned}
& \frac{\partial S M}{\partial \tan \varphi}=\frac{1}{\sin \theta}\left[\frac{1}{2} \gamma H^{2} \frac{\sin (\beta-\theta)}{\sin \beta} \cos \theta\right] \\
& \frac{\partial S M}{\partial \gamma}=\frac{1}{\sin \theta}\left[\frac{1}{2} H^{2} \frac{\sin (\beta-\theta)}{\sin \beta}(\cos \theta \tan \varphi-\sin \theta)\right] \\
& u(S F)=\sqrt{\left(\frac{\partial S F}{\partial c}\right)^{2} u_{c}^{2}+\left(\frac{\partial S F}{\partial \tan \varphi}\right)^{2} u_{\text {tan } \varphi}^{2}+\left(\frac{\partial S F}{\partial \gamma}\right)^{2} u_{\gamma}^{2}} \\
& \frac{\partial S F}{\partial c}=\frac{2}{\gamma} \frac{1}{H \sin (\beta-\theta) \sin \theta / \sin \beta}
\end{aligned}
$$

$$
\begin{aligned}
& \frac{\partial S F}{\partial \tan \varphi}=\frac{1}{\tan \theta} \\
& \frac{\partial S F}{\partial \gamma}=-\frac{2 c}{\gamma^{2}} \frac{1}{H \sin (\beta-\theta) \sin \theta / \sin \beta} \\
& S F=[2 \mathrm{c} / \gamma+H \sin (\beta-\theta)(\cos \theta \tan \varphi) / \sin \beta] /[H \sin (\beta-\theta) \sin \theta / \sin \beta]
\end{aligned}
$$

A slope geometry example of $\beta=60^{\circ}$ and $H=25 \mathrm{~m}$ and the material properties from Tables 5 and 6 are considered to exhibit the safety probability calculation. A deterministic calculation of the best estimate of the mean of $S M_{m}$ and $S F_{m}$ from Eqs. $(28,29)$ respectively is computed first, by the best estimates of the mean of the soil properties (i.e. $c_{m}, \tan (\varphi)_{m}$ and $\left.\gamma_{m}\right)$. Then a normal distribution for $S M$ and $S F$ is applied, in order to estimate their value for a probability not greater than $5 \%$ (i.e., Eq.(38) with $k=1.64485)$. The uncertainties $u(S M)$ and $u(S F)$ are calculated by Eqs.(30,34) applying the best estimates of the mean and the corresponding uncertainties of $c, \tan (\varphi)$ and $\gamma$ (error propagation considers the standard error of the mean, i.e. $u=S E$ ). The values of $S M$ and $S F$ for probability $p=5 \%$ and the corresponding probability $p$ for $S M<0$ and $S F<0$, all calculated for the critical plane $\theta_{c r}$ that gives the minimum $S M_{m}$, are presented in Tables 7 and 8 . The two sets of statistical measures from Table 5 for soil strength properties have been used.

$$
S M=S M_{m}-k \cdot u(S M), S F=S F_{m}-k \cdot u(S F)
$$

\begin{tabular}{cccccccccc}
\hline $\begin{array}{c}c_{m} \\
(\mathrm{kPa})\end{array}$ & $(\tan \varphi)_{m}$ & $\begin{array}{c}u_{c} \\
(\mathrm{kPa})\end{array}$ & $u_{\text {tan } \varphi}$ & $\begin{array}{c}S M_{m}= \\
\min \left(S M_{m}\right) \\
(\mathrm{kPa})\end{array}$ & $\begin{array}{c}\theta_{c r} \\
(\circ)\end{array}$ & $\begin{array}{c}u_{S M} \\
(\mathrm{kPa})\end{array}$ & $\begin{array}{c}S M_{p=5 \%}= \\
S M_{m}-k u_{S M} \\
(\mathrm{kPa})\end{array}$ & $V_{S M}$ & $\begin{array}{c}p_{(\mathrm{SM}<0)} \\
(\%)\end{array}$ \\
\hline 66.00 & 0.58225 & 15.46 & 0.03470 & 1435.08 & 48 & 527.49 & 567.43 & 2.721 & 0.33 \\
64.34 & 0.59415 & 18.56 & 0.04526 & 1396.92 & 48 & 631.85 & 357.62 & 2.211 & 1.35 \\
\hline
\end{tabular}

Table 7: Calculations of $S M$ for $p=5 \%$ and $p$ for $S M<0$ based on the minimum best estimate of the $S M_{m}$ and the corresponding $\theta_{c r}$

\begin{tabular}{cccccccccc}
\hline $\begin{array}{c}c_{m} \\
(\mathrm{kPa})\end{array}$ & $(\tan \varphi)_{m}$ & $\begin{array}{c}u_{c} \\
(\mathrm{kPa})\end{array}$ & $u_{\text {tan } \varphi}$ & $\begin{array}{c}S F_{m}= \\
\min \left(S F_{m}\right) \\
(\mathrm{kPa})\end{array}$ & $\begin{array}{c}\theta_{c r} \\
(\circ)\end{array}$ & $\begin{array}{c}u_{S F} \\
(\mathrm{kPa})\end{array}$ & $\begin{array}{c}S F_{p=5 \%}=S F_{m} \\
-k u_{S F} \\
(\mathrm{kPa})\end{array}$ & $V_{S M}$ & $\begin{array}{c}p_{(\mathrm{SF}<1)} \\
(\%)\end{array}$ \\
66.00 & 0.58225 & 15.46 & 0.03470 & 1.639 & 40 & 0.241 & 1.243 & 6.800 & 0.40 \\
64.34 & 0.59415 & 18.56 & 0.04526 & 1.6300 & 40 & 0.284 & 1.163 & 5.740 & 1.33 \\
\hline
\end{tabular}

Table 8: Calculations of $S F$ for $p=5 \%$ and $p$ for $S F<1$ based on the minimum best estimate of the $S F_{m}$ and the corresponding $\theta_{c r}$. 
It is obvious that the minimum values of the estimated means $S M_{m}=1435.08 \mathrm{kPa}$ and $S F_{m}=1.639$ develop for different critical angle $\theta_{c r}$ of the plane of failure. However, the corresponding values of $S M_{p=5 \%}$ and $S F_{p=5 \%}$ (calculated in Tables 7 and 8 by applying Eq.(38)) are not the minimum ones, nor are $p_{S M<0}$ and $p_{S F<1}$ the maximum ones, as their minimum and maximum values develop for different critical slopes $\theta_{c r}$ (presented in Tables 9 and 10). The differences for the problem examined are of the order of $4 \%$ for the $S M$ with respect to the $\min \left(S M_{p=5 \%}\right)$ value and of the order of 1 to $2 \%$ for the $S F$ with respect to the $\min \left(S F_{p=5 \%}\right)$.

\begin{tabular}{cccc|cccccc}
\hline & & & & \multicolumn{5}{|c}{ Calculations based on min $S M$ for $p=5 \%$} \\
\hdashline$c_{m}$ & $(\tan \varphi)_{m}$ & $\begin{array}{c}u_{c} \\
(\mathrm{kPa})\end{array}$ & $u_{\text {tan } \varphi}$ & $\begin{array}{c}\min \left(S M_{p=5}\right) \\
(\mathrm{kPa})\end{array}$ & $\begin{array}{c}\theta_{c r} \\
(\mathrm{o})\end{array}$ & $\begin{array}{c}u_{S M} \\
(\mathrm{kPa})\end{array}$ & $\begin{array}{c}S M_{\mathrm{m}} \\
(\mathrm{kPa})\end{array}$ & $V_{S M}$ & $p_{S M<0}$ \\
\hline 66.00 & 0.58225 & 15.46 & 0.03470 & 554.18 & 46 & 546.51 & 1453.11 & 2.659 & 0.39 \\
64.34 & 0.59415 & 18.56 & 0.04526 & 340.75 & 46 & 654.42 & 1417.49 & 2.165 & 1.54 \\
\hline
\end{tabular}

Table 9.1: Calculated minimum $S M$ for $p=5 \%$ and the corresponding $\theta_{c r}, u_{S M}$ and $S M_{m}$ values.

\begin{tabular}{|c|c|c|c|c|c|c|c|c|c|}
\hline \multirow[b]{2}{*}{$\begin{array}{c}c_{m} \\
(\mathrm{kPa})\end{array}$} & \multirow[b]{2}{*}{$(\tan \varphi)_{m}$} & \multirow[b]{2}{*}{$\begin{array}{c}u_{c} \\
(\mathrm{kPa})\end{array}$} & \multirow[b]{2}{*}{$u_{\tan \varphi}$} & \multicolumn{6}{|c|}{ Calculations based on max $p$ for $S M<0$} \\
\hline & & & & $\begin{array}{c}\max \left(p_{\mathrm{SM}<0)}\right. \\
(\%)\end{array}$ & $\begin{array}{l}\theta_{c r} \\
(\mathrm{o})\end{array}$ & $\begin{array}{c}u_{S M} \\
(\mathrm{kPa})\end{array}$ & $\begin{array}{l}S M_{m} \\
(\mathrm{kPa})\end{array}$ & $V_{S M}$ & $\begin{array}{c}S M_{p=5 \%} \\
(\mathrm{kPa})\end{array}$ \\
\hline 66.00 & 0.58225 & 15.46 & 0.03470 & 0.40 & 45 & 556.72 & 1475.12 & 2.650 & 559.40 \\
\hline 64.34 & 0.59415 & 18.56 & 0.04526 & 1.54 & 45 & 667.07 & 1440.88 & 2.160 & 343.64 \\
\hline
\end{tabular}

Table 9.2: Calculated maximum $p$ for $S M<0$ and the corresponding $\theta_{c r}, u_{S M}$ and $S M_{m}$ values.

\begin{tabular}{|c|c|c|c|c|c|c|c|c|c|}
\hline \multirow[b]{2}{*}{$\begin{array}{c}c_{m} \\
(\mathrm{kPa})\end{array}$} & \multirow[b]{2}{*}{$(\tan \varphi)_{m}$} & \multirow[b]{2}{*}{$\begin{array}{c}u_{c} \\
(\mathrm{kPa})\end{array}$} & \multirow[b]{2}{*}{$u_{\tan \varphi}$} & \multicolumn{6}{|c|}{ Calculations based on min SF for $p=5 \%$} \\
\hline & & & & $\begin{array}{c}\min \left(S F_{p=5 \%}\right) \\
(\mathrm{kPa})\end{array}$ & $\begin{array}{l}\theta_{c r} \\
(\circ)\end{array}$ & $\begin{array}{c}\text { USF }_{S} \\
(\mathrm{kPa})\end{array}$ & $\begin{array}{l}S F_{\mathrm{m}} \\
(\mathrm{kPa})\end{array}$ & $V_{S F}$ & $p_{S F<0}$ \\
\hline 66.00 & 0.58225 & 15.46 & 0.03470 & 1.231 & 42.5 & 0.260 & 1.659 & 6.383 & 0.56 \\
\hline 64.34 & 0.59415 & 18.56 & 0.04526 & 1.141 & 43.5 & 0.317 & 1.662 & 5.243 & 1.83 \\
\hline
\end{tabular}

Table 10.1: Calculated minimum $S F$ for $p=5 \%$ and the corresponding $\theta_{c,}, u_{S F}$ and $S F_{m}$ values.

\begin{tabular}{|c|c|c|c|c|c|c|c|c|c|}
\hline \multirow[b]{2}{*}{$\begin{array}{c}c_{m} \\
(\mathrm{kPa})\end{array}$} & \multirow[b]{2}{*}{$(\tan \varphi)_{m}$} & \multirow[b]{2}{*}{$\begin{array}{c}u_{c} \\
(\mathrm{kPa})\end{array}$} & \multirow[b]{2}{*}{$U_{\tan \varphi}$} & \multicolumn{6}{|c|}{ Calculations based on max $p$ for $S F<0$} \\
\hline & & & & $\begin{array}{c}\max \left(p_{\mathrm{SF}<0}\right) \\
(\%)\end{array}$ & $\begin{array}{l}\theta_{c r} \\
\left({ }^{\circ}\right)\end{array}$ & $\begin{array}{c}u_{S F} \\
(\mathrm{kPa})\end{array}$ & $\begin{array}{c}S F_{m} \\
(\mathrm{kPa})\end{array}$ & $V_{S F}$ & $\begin{array}{c}S F_{p=5 \%} \\
(\mathrm{kPa})\end{array}$ \\
\hline 66.00 & 0.58225 & 15.46 & 0.03470 & 0.62 & 45 & 0.287 & 1.718 & 5.976 & 1.245 \\
\hline 64.34 & 0.59415 & 18.56 & 0.04526 & 1.90 & 45 & 0.338 & 1.701 & 5.035 & 1.145 \\
\hline
\end{tabular}

Table 10.2: Calculated maximum $p$ for $S F<0$ and the corresponding $\theta_{c r}, u_{S F}$ and $S F_{m}$ values..

The second set of soil strength parameters lead to more conservative results. The comparison of the results of Tables 9.1 and 9.2 with Table 7 and of Tables 10.1 and 10.2 with Table 8 shows that for the probabilistic analyses it is preferable to determine the critical surface that corresponds to the minimum calculated $S M$ or $S F$ for a probability of exceedance $5 \%$ (or $90 \%$ confidence level, i.e. $\min S M_{p=5 \%}$ or $\min S F_{p=5 \%}$ ), instead of calculating the critical surface of the minimum mean value $S M_{m}$ or $S F_{m}$ first and then the corresponding $S M_{p=5 \%}$ or $S F_{p=5 \%}$. This happens because there is no linear relationship between $S M_{m}$ (or $S F_{m}$ ) and $u_{S M}$ (or $u_{S F}$ ) for a monotonically increasing or reducing failure plane angle.

Concerning the influence of the individual uncertainties Fig.9 shows their influence on the minimum $S M$ and Fig.10 on the probability of having an $S M<0$. Fig.11 shows their influence on the minimum $S F$ and Fig. 12 on the probability of having an $S F<1$. It is apparent that for this specific problem, the most influential factor on the probabilistic $S M$ or $S F$ is the uncertainty of cohesion. This is important because cohesion generally has a greater uncertainty from the angle of shearing resistance. The dependence $S M$ and $S F$ with the various uncertainties present the same trends.

The above results will now be compared with the results from the deterministic analysis, for which the application of Eurocode 7 has been considered and more specifically the design analysis 3 (DA-3). DA-3 has become the national choice 
of many countries for the case of overall stability of natural and cut slopes. According to this, for the drained conditions, the following components are used for deterministic analysis: a) the design strength $c_{d}=c_{k} /\left(\gamma_{M} \gamma_{m}\right),(\tan \varphi)_{d}=(\tan \varphi)_{k} /\left(\gamma_{M} \gamma_{m}\right)$ with $\gamma_{m}=1.0$ (irrespectively of the type of analysis) and $\gamma_{M}=1.25$, b) the permanent actions $G_{d}=\gamma_{G} G_{k}$, with $\gamma_{G}=1.0$ and c) the mobile design loads are $Q_{d}=\gamma_{2} Q_{k}$, with $\gamma_{Q}=0$ or 1.3 for favourable or unfavourable loads respectively.

Table 11 shows the results for the deterministic analyses, in which the four characteristic strength values of Table 6 have been used, factored by $\gamma_{M}=1.25$ for the design strength values. The minimum safety margin $(S M)$ and safety factor $(S F)$
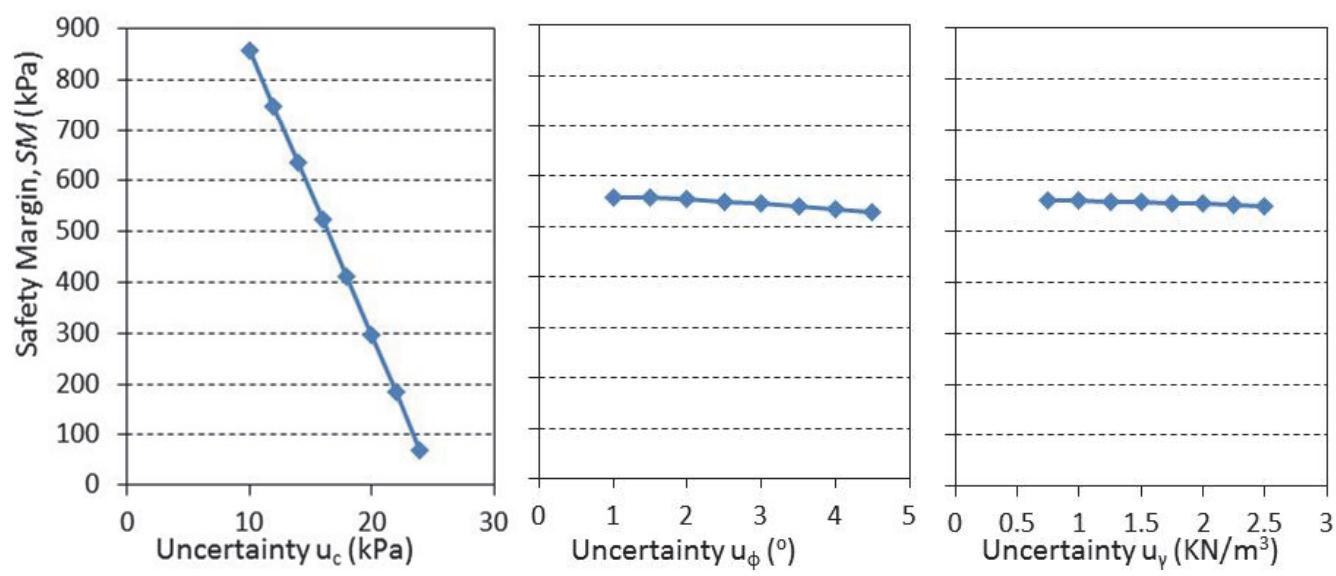

Figure 9: Influence of each uncertainty coefficient on the value of $S M=S M_{m}-k u_{S M}$.
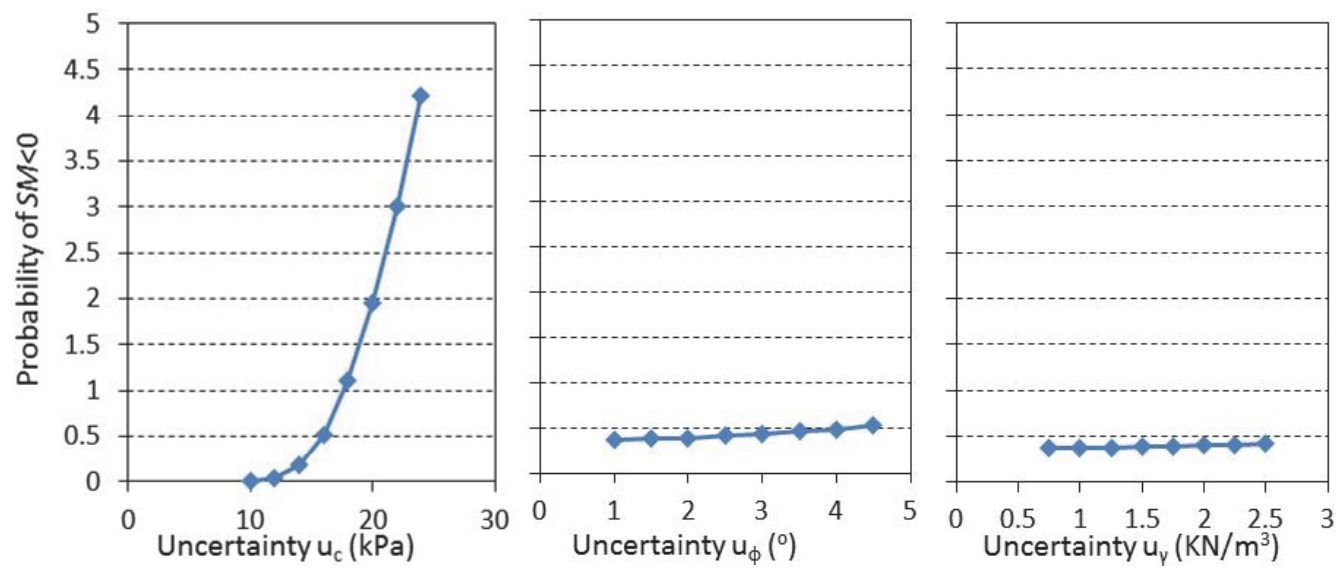

Figure 10: Influence of each uncertainty coefficient on the probability of $S M<0$.
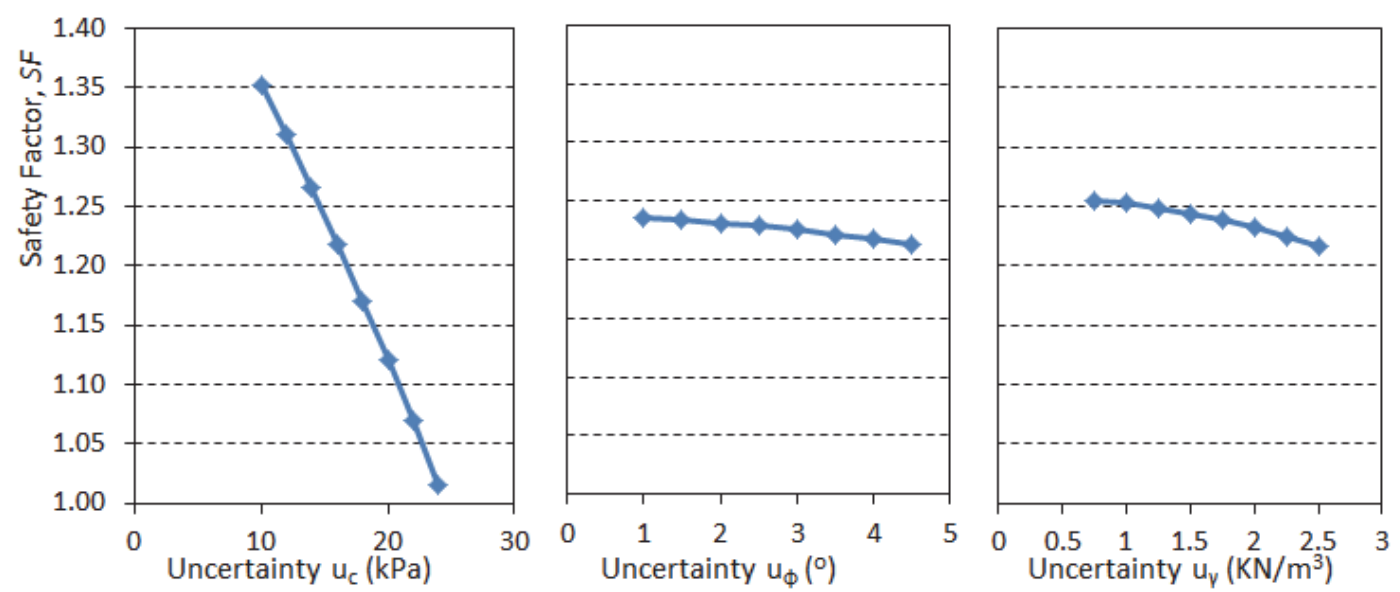

Figure 11: Influence of each uncertainty coefficient on the value of $S F=S F_{m}-k u_{S F}$. 

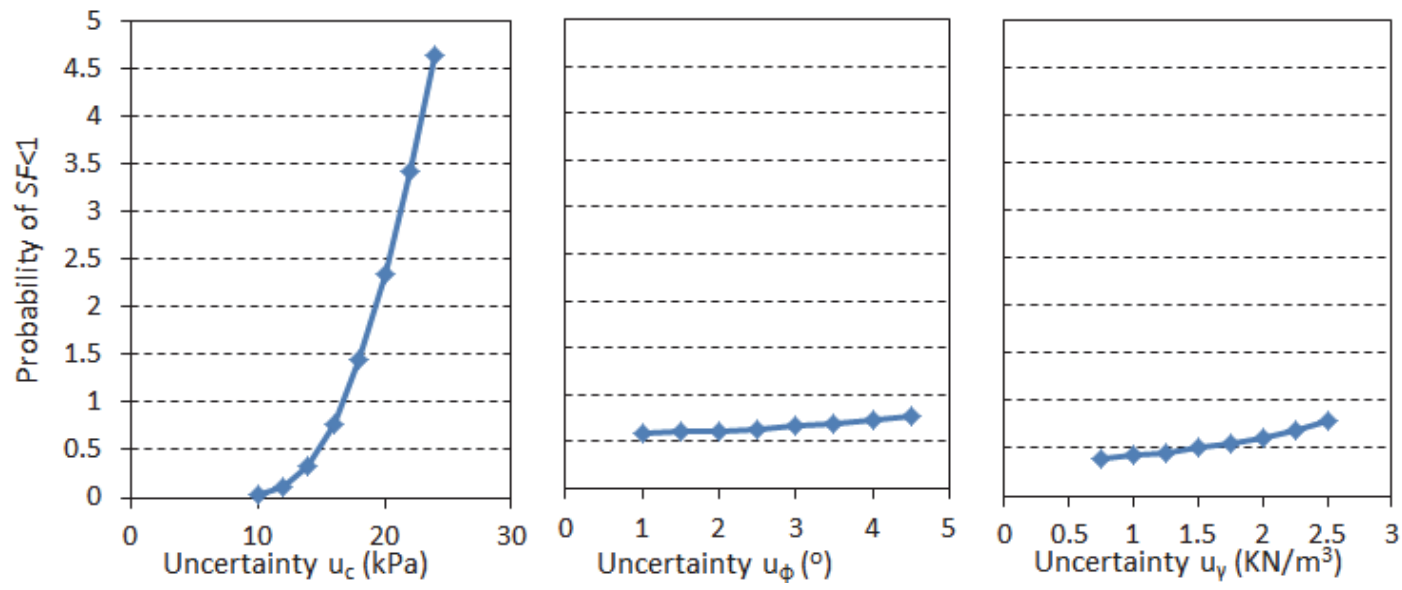

Figure 12: Influence of each uncertainty coefficient on the probability of $S F<1$.

and their corresponding critical slip surface $\left(\theta_{c r}\right)$ are presented. The material unit weight has been taken $\gamma_{s i l, m}=22 \mathrm{kN} / \mathrm{m} 3$ with uncertainty $u_{\gamma}=2 \mathrm{kN} / \mathrm{m}^{3}$. According to the calculations, only characteristic 2 gives acceptable safety margin $(S M>0)$ and safety factor $(S F>1.00)$ and. The critical failure plane determined by $S M$ and $S F$ do not coincide. Characteristic values 1 and 4 were derived from the statistical measures used in the above analyses. It is obvious that probabilistic and deterministic analyses can give the opposite result. In our case the deterministic analysis was more conservative, which has to do with the selection of the partial safety factors.

\begin{tabular}{ccccccccc}
\hline$i$ & $c_{k i}(\mathrm{kPa})$ & $\tan \varphi_{k i}$ & $c_{d i}(\mathrm{kPa})$ & $\tan \varphi_{d i}$ & $S M_{i}$ & $\theta_{c, S M, i}\left({ }^{\circ}\right)$ & $F S_{i}$ & $\theta_{c, F S, i}\left({ }^{\circ}\right)$ \\
1 & 39.60 & 0.52297 & 31.38 & 0.41837 & -133.35 & 41 & 0.947 & 42 \\
2 & 55.65 & 0.56288 & 44.52 & 0.45030 & 431.74 & 43 & 1.174 & 40.5 \\
3 & 43.43 & 0.49395 & 34.74 & 0.39516 & -86.60 & 40.5 & 0.967 & 41 \\
4 & 29.82 & 0.50999 & 23.86 & 0.40799 & -466.99 & 40 & 0.814 & 43.5 \\
\hline
\end{tabular}

Table 11: Deterministic stability calculation with design parameters..

\section{CONCLUSIONS}

$\mathrm{R}$

Reliability methods can be incorporated for the determination of soil strength statistical measures and for probabilistic limit equilibrium analyses. Regarding soil strength, the determination of the uncertainties of the Mohr - Coulomb failure criterion constants $c$ and $\tan \varphi$ from results of the typical triaxial test can be calculated through an error propagation technique, such as the FORM, applied to an appropriate performance function. This function relates the measured $\left(\sigma_{1}\right)$ and applied $\left(\sigma_{3}\right)$ quantities to the material constants $(c, \varphi)$ of the M-C failure criterion by a nonlinear relationship. The FORM was applied to triaxial data and the results from different approaches for calculating the best estimate of the mean, the uncertainties and the characteristic values of the $\mathrm{M}-\mathrm{C}$ constants were compared. For the approaches considered, the direct application of the FORM gave a lower uncertainty, since the consideration of each single specimen as independent increased the sample size. The uncertainties together with the corresponding best estimates were also used to estimate the characteristic failure envelope. Regarding the characteristic failure, envelope four different approaches were presented with different results. The FORM gave the more reliable results. In any case, engineering judgement on the results is necessary.

The results were applied to a simple planar failure problem, which allows for a direct comparison of the various statistical measures of the $S M$ and $S F$, since a single failure surface is considered. It was observed that the maximum probability of having a $S M<0$ or $S F<1$ does not correspond to the minimum best estimate of the $S M$ or $S F$. This is influenced by the non - linear relationship of $S M$ and $u_{S M}$ (or $S F$ and $u_{S F}$ ) function and needs further exploring. The sensitivity analysis showed that the only influential uncertainty was that of cohesion, which generally happens to have the greater variability with regards to other soil constants. A sensitivity analysis is recommended in probabilistic analyses in order to determine the influence of each uncertainty separately. 
The deterministic analyses gave in general more conservative results and only one gave acceptable $S M$ and $S F$ values. These analyses greatly depend on the selection of the partial safety factor selection that determines the characteristic value. The partial factors are indirectly related to an empirical probability of exceedance and the confidence of material properties and method of analysis. On the contrary, all probabilistic analyses gave acceptable $S M$ and $S F$ values, which means that probabilistic analyses could be used for an appropriate selection of partial safety factors. Therefore, the application of statistical methods can also set a framework for the selection of the characteristic mechanical properties, for deterministic analyses.

\section{REFERENCES}

[1] Baecher G. and Christian J. (2003). Reliability and Statistics in Geotechnical Engineering, Wiley, p. 618.

[2] Orr T. and Breysse D. (2008). Eurocode 7 and reliability-based design. Reliability-Based Design in Geotechnical Engineering. Computations and Applications, Kok-Kwang Phoon(edr), Taylor \& Francis, pp. $298-343$.

[3] ISO/IEC Guide 98-3:2008. Uncertainty of measurement - Part 3: Guide to the expression of uncertainty in measurement (GUM: 1995).

[4] Kulhawy H. (1992). On the evaluation of static soil properties, Stability and Performance of Slopes and Embankments - II, Proceedings of a Specialty Conference, Seed R.B. and Boulanger R.W. (eds), ASCE, pp. 95-115.

[5] Duncan J. M. (2000). Factors of safety and reliability in geotechnical engineering, Journal of Geotechnical and Geoenvironmental Engineering, 126(4), pp. 307-316.

[6] Schneider H.R. and Fitze P. (2013). Characteristic shear strength values for EC7: Guidelines based on a statistical framework. Proc. 15 th European Conference on Soil Mechanics and Geotechnical Engineering, 4, pp. 318-324.

[7] Fellin W. (2005). Assessment of characteristic shear strength parameters of soil and its implication in geotechnical design, Analyzing Uncertainty in Civil Engineering, Fellin, W., Lessmann, H., Oberguggenberger, M., Vieider, R. (eds.), Springer, pp. 1-15.

[8] Pohl C. (2011). Determination of characteristic soil values by statistical methods. ISGSR 2011 - Vogt, Schuppener, Straub \& Bräu (eds), pp. 427-434.

[9] Frank R., Bauduin C., Driscoll R., Kavvadas M., Krebs Ovensen N., Orr T. and Schuppener B. (2004). Designers' Guide to EN 1997-1 Eurocode 7: Geotechnical Design - General Rules, Thomas Telford, p. 216.

[10] Kottegoda N. T. and Rosso R. (2008). Applied Statistics for Civil and Environmental Engineers, 2nd Edition, WileyBlackwell, p. 736.

[11] Tsiambaos G. (1988). Technicogeological features of the Herakleion Crete Marls. PhD, University of Patras, p. 358 (in Greek).

[12] Schneider H.R. (1997). Definition and determination of characteristic soil properties. Proc. 14th International Conference on Soil Mechanics and Foundation Engineering, 4, pp. 2271-2274.

[13] Wu T. (2008). Reliability analysis of slopes, Reliability-Based Design in Geotechnical Engineering, Computations and Applications, Kok-Kwang Phoon (ed.), Taylor \& Francis, pp. 413-447. 